\title{
Model reduction of travelling-wave problems via Radon cumulative distribution transform
}

\author{
Jie Ren, ${ }^{1}$ William R. Wolf, ${ }^{2}$ and Xuerui $\mathrm{Mao}^{1,}{ }^{\text {* }}$ \\ ${ }^{1}$ Department of Mechanical Engineering, Faculty of Engineering, \\ University of Nottingham, Nottingham, NG7 2RD \\ ${ }^{2}$ School of Mechanical Engineering, Universidade Estadual de Campinas, Campinas, SP 13083-860, Brazil
}

(Dated: August 5, 2021)

\begin{abstract}
Travelling-wave problems, due to their sizeable Kolmogorov n-width, have brought critical challenges to conventional model reduction techniques. This study aims to provide new insights into this problem by exploiting the Radon cumulative distribution transform (R-CDT) [1] emerged in the sector of computer vision science. The core lies in the unique property of the nonlinear invertible R-CDT that renders both travelling and scaling components into amplitude modulations. In contrast to the physical space, a substantial model-reduction is achieved in the R-CDT space while sustaining high accuracy. The method is parameter-free and data-driven that lends itself to problems regardless of the dimensions or boundary conditions. Examples start with one-dimensional Burger's equation subject to non-periodic boundary conditions, where both travelling and diffusion dominate the physics. Further in higher-dimensional problems, we show the model reduction of travelling Gaussian solitons. In addition to foreseeable motions, the proposed method is capable of handling random travelling with a non-differentiable trajectory.
\end{abstract}

\section{INTRODUCTION}

Model reduction of physical data, either simulated or measured, plays a significant role in understanding the concealed mechanisms while granting plentiful downstream applications, e.g. data compression, model-baseds control and predictions [2. The reduction has been backed by the fact that the inherent physics is usually sparse in the degree of freedom [3] despite the bulky size of the generated data. However, with standard model reduction techniques, e.g. proper orthogonal decomposition (POD) and dynamic mode decomposition (DMD), the accuracy and efficiency immensely rely on the narrowness of the data's Kolmogorov n-width, of which travelling-wave problems fail to minimise.

There has been a growing interest in advancing model reduction techniques for travelling-wave problems. In tableI we compare some recently developed methods. Notably, a direct thought dates back to the shifted-based templatefitting method [4, in which the data can be properly shifted in space such that a travelling wave appears to be stationary in the transformed coordinates. To achieve adequate model reduction and diminish the loss of accuracy, this type of method will require to determine the appropriate shifting matrix and introduce algorithms to minimise the error.

The optimal shift matrix recently becomes addressed in an optimisation framework developed by Mendible et al. [5]. The named method, unsupervised travelling wave identification with shifting and truncation (UnTWIST), seeks the analytical representations of multiple travelling velocities by combining ridge detection, spectral clustering and sparse relaxed regularised regression. A physically precise shift is thus made possible for each travelling wave carried by the system. Besides algorithms recognising the transport velocities, shifted-POD [6] provides a pioneering approach accounting for the post-shift stage, in which the data-shifting introduces errors when multiple different waves are

TABLE I. A comparison of recently developed dimensionality-reduction methods for travelling-wave problems.

\begin{tabular}{ccc}
\hline \hline Methods & Spatial dimension & Boundary condition \\
\hline UnTWIST [5] & $1-\mathrm{D}$ & Periodic \\
Shifted-POD [6] & $1-\mathrm{D}, 2-\mathrm{D}$ & Periodic \\
cDMD [7] & $1-\mathrm{D}, 2-\mathrm{D}$ & Periodic \\
Transport Reversal [8] & $1-\mathrm{D}$ but extendable & Any \\
Data calibration [9] & $1-\mathrm{D}$ & Periodic \\
TSMOR [10] & $1-\mathrm{D}, 2-\mathrm{D}$ & Any \\
AMD [1] & $1-\mathrm{D}, 2-\mathrm{D}$ & Any \\
AADEIM [12] & $1-\mathrm{D}, 2-\mathrm{D}, 3-\mathrm{D}$ & Any \\
Radon-CDT (present method) & $1-\mathrm{D}, 2-\mathrm{D}, 3-\mathrm{D}$ & Any \\
\hline \hline
\end{tabular}


present. Shifted-POD iteratively minimises the residual between the data snapshot and its POD approximation with a least-squares optimisation, capable of achieving machine precision. In line with the idea of shift-based methods, characteristic DMD (cDMD) [7] rotates the coordinate system both in space and time. The rotating angle is determined by the group velocity of the travelling waves, such that the physics in the new frame develops along the characteristic line, supporting a significant singular value drop.

However, shift-based methods confront data loss unless the boundary locks up the travelling waves with periodic or reflecting conditions. Transport Reversal $[8$ generalised the template fitting approach 4 ] by introducing a set of enhancement algorithms (e.g. Greedy iteration, cut-off vectors). The method thus manages more general problems (with varying shapes, non-periodic boundary conditions).

Another prospect to model travelling-wave problems is through a pre-conditioning operation. Data Calibration [9] makes use of an invertible mapping to calibrate the data, where the Kolmogorov n-width becomes reduced such that the required modes are much less. The method has been developed in the 1-D framework with periodic boundary conditions. The proposed transform still requires a priori expertise on the behaviour of the physical data.

Apart from works described above, various new or improved model reduction methods have been proposed. For example, transported snapshot model order reduction (TSMOR) [10 is specially designed to handle problems with moving shocks (discontinuities). The transports are approximated as a low-order polynomial expansion. Advection mode decomposition (AMD) [1] defines an advection-mode hierarchy based on Wasserstein distance to separate advection feature of the flow while the residuals are decomposed with standard POD. Peherstorfer [12] proposed adaptive bases and adaptive sampling discrete empirical interpolation method (AADEIM), exploiting the temporal and spatial locality of convective structures. The local basis gets updated online following an adaptive sampling scheme querying the full model at a few selected spatial coordinates.

While the above-reviewed researches handle travelling-wave problems in various aspects, assessment on crucial properties (e.g. problem dimension, boundary condition as tabulated in table II) shows that the model reduction towards practical applications is far from mature. In particular, a majority of the methods are not ready for threedimensional problems. Some methods are target-oriented and require essential knowledge of the physical problem to be known in advance, while shift-based methods put restrictions on boundary conditions. In this study, we aim to boost the effort with ideas generated in the sector of computer vision science. We propose to build reducedorder models in the Radon cumulative distribution transformed (R-CDT) space, such that the Kolmogorov n-width becomes significantly reduced. The method solves multi-dimensional problems and possesses flexibility with boundary conditions. It is also parameter-free and explicit, thus training as generally required by machine-learning [13, is not necessary. The rest of the letter begins by introducing the methodology followed by examples with increasing dimensions.

\section{METHODOLOGY}

The heart of the proposed R-CDT space is the cumulative distribution transform (CDT) [14] while Radon transform is applied to break higher-dimensional problems into a series of 1-D signals. CDT interprets 1-D signals as probability density functions. To meet this requirement, a given input signal $f(x)$ and a reference signal $r(x)$, both defined on $\left[x_{1}, x_{2}\right]$, are firstly normalised such that

$$
\int_{x_{1}}^{x_{2}} f(x) \mathrm{d} x=\int_{x_{1}}^{x_{2}} r(x) \mathrm{d} x=1, \text { with } f(x)>0, r(x)>0 .
$$

The forward CDT of $f(x)$ with respect to $r(x)$ is defined by the strictly increasing function $\hat{f}(x)$ that satisfies

$$
\int_{x_{1}}^{\hat{f}(x)} f\left(x^{\prime}\right) \mathrm{d} x^{\prime}=\int_{x_{1}}^{x} r\left(x^{\prime}\right) \mathrm{d} x^{\prime} .
$$

The inverse of CDT (iCDT) is obtained by differentiating (2) with respect to $x$ :

$$
f(x)=r\left(\hat{f}^{-1}(x)\right) \frac{\mathrm{d}}{\mathrm{d} x} \hat{f}^{-1}(x), \text { where } \hat{f}^{-1}(\hat{f}(x))=x .
$$

A denormalisation step is applied to bring the signal to its original range. CDT establishes a nonlinear invertible oneto-one mapping between the signal and its transformation, and therefore, retains all the information contained in the original signal. The advantage of CDT over the other linear transforms, e.g. Fourier transform, wavelet transform, lie 
$(a)$

$(c)$
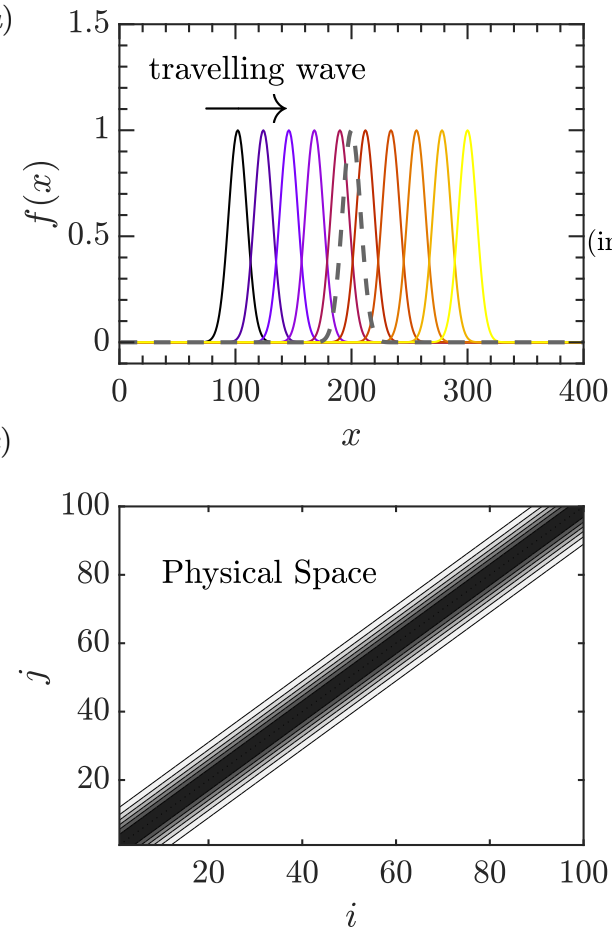

$(b)$

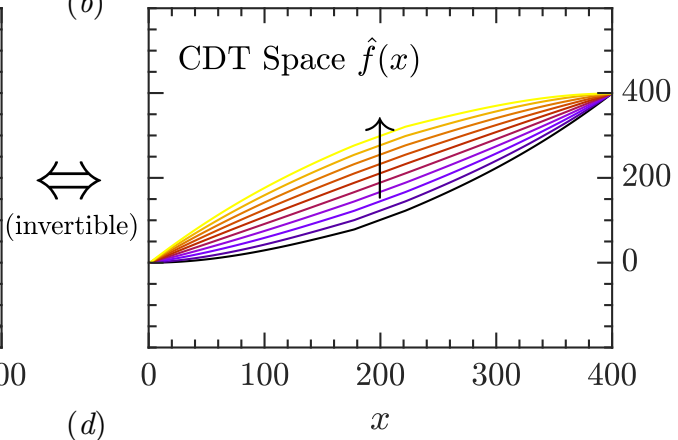

(d)

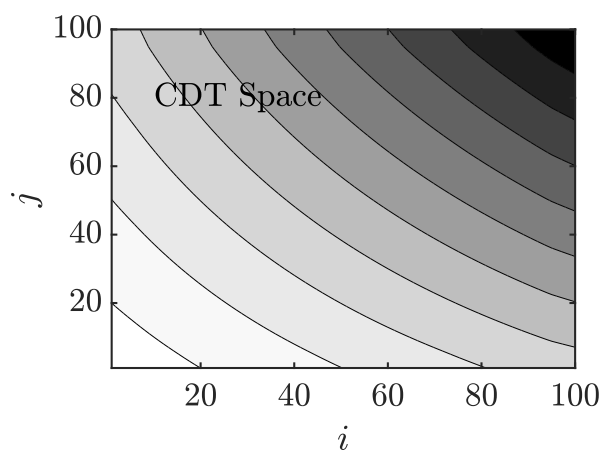

FIG. 1. Travelling Gaussian soliton $f(x, t)$ (a) and its CDT transform $\hat{f}(x, t)$ (b). Solid lines are coloured by time travelled, while the dashed line indicates the template. The pseudo-colour plot of the correlation matrix $\boldsymbol{R}_{i j}=\left\langle f\left(x, t_{i}\right), f\left(x, t_{j}\right)\right\rangle$ in physical (c) and CDT (d) space.

in its exclusive composition properties that render travelling and scaling components into amplitude modulations. For example, the CDT of a travelling signal $f(\alpha x-c t)$ with phase velocity $c$ and scaling factor $\alpha$ is given by $(\hat{f}(x)+c t) / \alpha$. A proof is given in Appendix.

Figure 1 demonstrates this property. The travelling wave (coloured by the time travelled in figure 1 (a)) converts to a purely stationary signal with growing amplitude in the CDT space shown in figure 1(b). In figure 1(c,d), we examine the correlation matrix $\boldsymbol{R}_{i j}=\left\langle f\left(x, t_{i}\right), f\left(x, t_{j}\right)\right\rangle$. Generally speaking, a fast decay of eigenvalues of $\boldsymbol{R}$ warrants a narrow Kolmogorov n-width and an effective model-reduction. This decay is determined by the nonorthogonality of $\boldsymbol{R}$, which can be viewed as a deviation of $\boldsymbol{R}$ from the identity matrix (identity matrix gives rise to non-decaying eigenvalues). As shown in figure 1 (c, d), the diagonally dominant correlation matrix in the physical space becomes a globally distributed matrix in the CDT space (figure 1(d)), retaining a reduced Kolmogorov n-width. In the present study, we define the range of function $\hat{f}(x)$ to be the same as its domain, namely, $\hat{f}\left(x_{1}\right)=x_{1}, \hat{f}\left(x_{2}\right)=x_{2}$. This confinement will help to ensure the robustness and reversibility of CDT provided the signal is differentiable and properly normalised. From (2), $r(x)$ determines the shape of the transformed profile $\hat{f}(x)$. Nonetheless, it has been tested that different choices of $r(x)$, for example, an arbitrary snapshot of the input data at a chosen time step $t_{r}$, $r(x)=f\left(x, t_{r}\right)$ or standard analytic functions $r(x)=\sin (x)+2$, lead to similar performance of model reduction. In the examples showed below, results are obtained with $r(x)=f\left(x, t_{r}\right)$. CDT, in nature, has the same mathematical foundations as optimal transportation (OT) [15]. Here "optimal" lies in the minimal Wasserstein distance between the signal and template, defined as

$$
\|L\|_{2}=\left[\int_{x_{1}}^{x_{2}}(\hat{f}(x)-x)^{2} r(x) \mathrm{d} x\right]^{\frac{1}{2}}
$$

reflecting the geometric connotation of CDT.

For higher-dimensional problems, we apply Radon transform to the signal before CDT. Radon transform [see 16, for a comprehensive introduction] finds its roots in multidisciplinary sciences, including medical imaging, geophysics, material science, optics etc. The inverse transform (iRadon) represents the mathematical framework of reconstruction from probe-measured (e.g. by X rays, microwaves) distributions. Radon transform integrates an image or volume $f$ 
along different angles $(0 \leqslant \theta, \phi<\pi)$ leading to a number of projected profiles $\tilde{f}$, defined as

$$
\begin{cases}2-\mathrm{D}: & \tilde{f}(s, \theta)=\iint_{-\infty}^{+\infty} f(x, y) \delta(s-x \cos \theta-y \sin \theta) \mathrm{d} x \mathrm{~d} y \\ \text { 3-D: } & \tilde{f}(s, \theta, \phi)=\iiint_{-\infty}^{+\infty} f(x, y, z) \delta(s-x \sin \theta \cos \phi-y \sin \theta \sin \phi-z \cos \theta) \mathrm{d} x \mathrm{~d} y \mathrm{~d} z,\end{cases}
$$

and $\delta$ is the Dirac delta function. The Radon-CDT (R-CDT) transform $\hat{f}[1,17$ is then obtained by applying CDT along each projection angle:

$$
\int_{s_{1}}^{\hat{f}(s, \theta)} \tilde{f}\left(s^{\prime}, \theta\right) \mathrm{d} s^{\prime}=\int_{s_{1}}^{s} \tilde{r}\left(s^{\prime}, \theta\right) \mathrm{d} s^{\prime}, \int_{s_{1}}^{\hat{f}(s, \theta, \phi)} \tilde{f}\left(s^{\prime}, \theta, \phi\right) \mathrm{d} s^{\prime}=\int_{s_{1}}^{s} \tilde{r}\left(s^{\prime}, \theta, \phi\right) \mathrm{d} s^{\prime}
$$

where $\tilde{r}$ is the Radon transform of the template $r(x, y ; z)$. Below we show the properties of R-CDT for 2-D problems, while a similar relation holds for the 3-D framework. Following the definition (5), the Radon transform of a travelling signal $g(x, y)=f(\alpha x-b, \alpha y-c)$ is

$$
\tilde{g}(s, \theta)=\tilde{f}(\alpha s-\alpha b \cos \theta-\alpha c \sin \theta, \theta) .
$$

This implies that, the 2-D travelling $\boldsymbol{v}=(b, c)$ and scaling $\alpha$ in physical space becomes a 1-D traveling of $\alpha b \cos \theta+$ $\alpha c \sin \theta$ and scaling of $\alpha$ in the Radon space. From the composition properties of CDT, the following relation thus holds in the R-CDT space.

$$
\hat{g}(s, \theta)=\frac{1}{\alpha^{2}} \hat{f}(s, \theta)+\frac{b}{\alpha} \cos \theta+\frac{c}{\alpha} \sin \theta .
$$

In contrast to the appearance in physical space, $g(x, y)=f(\alpha x-b, \alpha y-c)$, the travelling and scaling component turns into amplitude modulations in the R-CDT space. Moreover, the application of 1D OT combined with Radon transform have been successful in solving multi-dimensional optimal transport problems with applications in image processing [18, model reduction of BGK equations [19] to name a few.

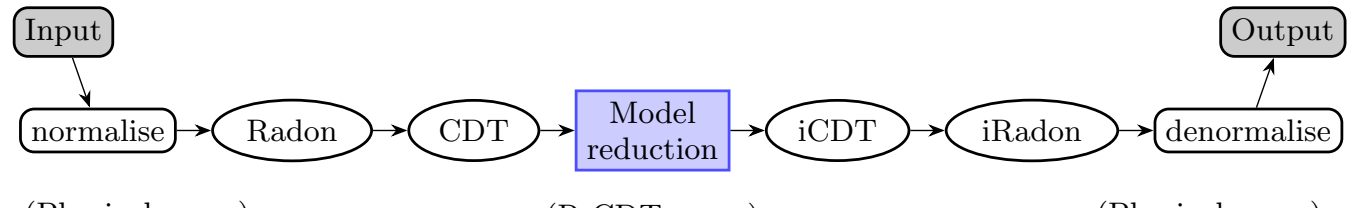
(Physical space)
(R-CDT space)
(Physical space)

FIG. 2. The flow-chart of the proposed method.

Figure 2 provides a work-flow summary of the proposed method. In examples presented in this article, we build the reduced-order model (ROM) in the R-CDT space by applying a standard POD. As both CDT and Radon transforms are invertible, the inverse transform (iRadon and iCDT) and denormalisation are then performed to reconstruct the physical space. When the data type is of vectorial nature, the reduced order models are built in the R-CDT space of each component respectively.

\section{EXAMPLES}

\section{The 1-D problem: Viscous Burgers' equation}

The viscous Burgers' equation is given by

$$
u_{t}+u u_{x}=\nu u_{x x} .
$$

As a model equation mimicking the formation of shock waves, the solution takes the form of diffusive waves that travel with speeds proportional to the wave height. Similar to the case described in Mendible et al. [5], we consider $\nu=0.1$, a Gaussian soliton, $u(x, t=0)=\exp \left(-(x+2)^{2}\right)$ is prescribed as an initial condition, we limit the domain 

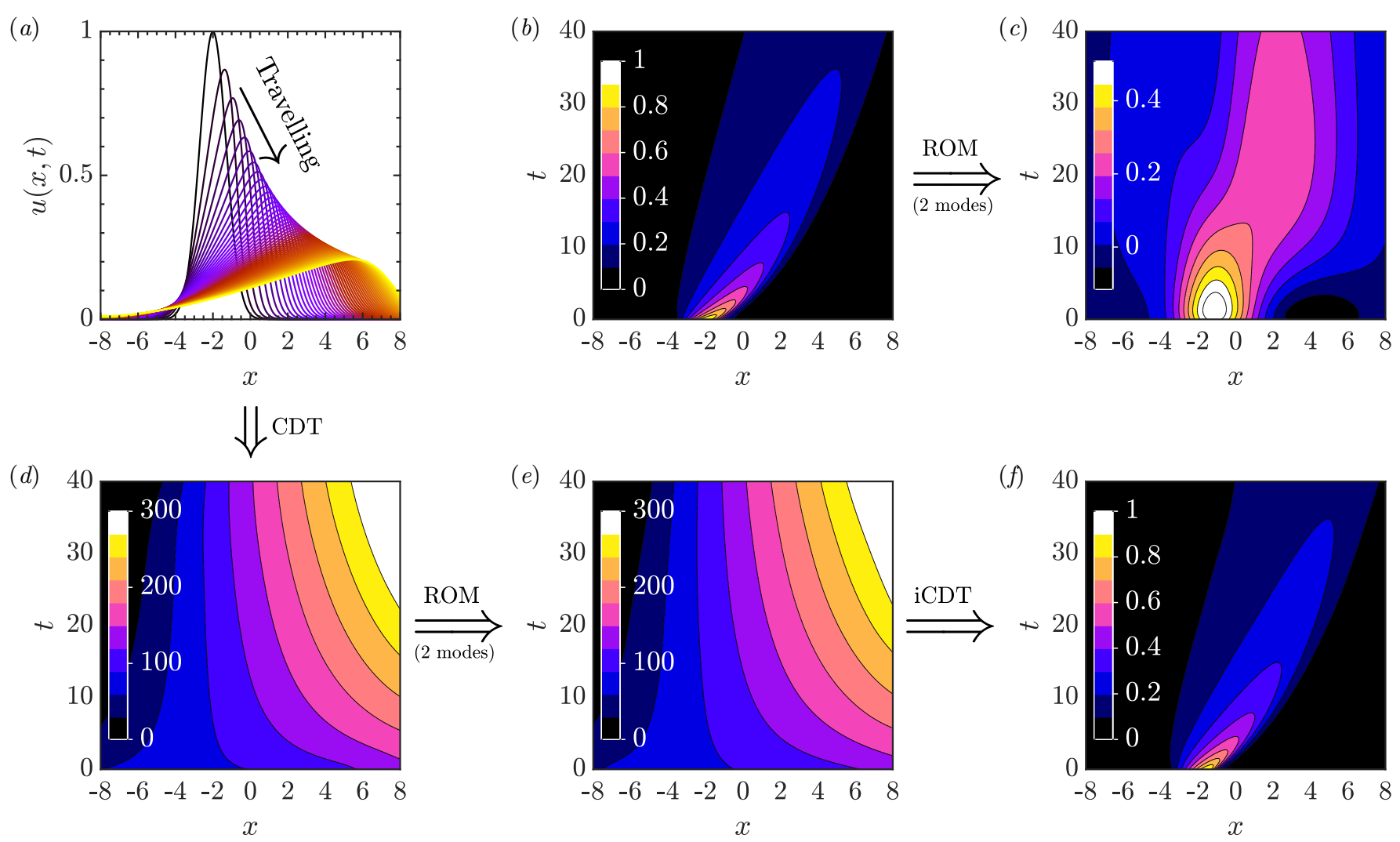

FIG. 3. Model reduction of the viscous Burgers' equation. The evolution of the wave profiles is visualised in panel (a), where the lines are coloured by the time travelled. We show the original signal and its 2-mode ROM in the $x-t$ frame in panel (b) and (c). With the proposed method, the physical signal is transformed into the CDT space (d), where the 2-mode ROM is built (e), this is followed by an iCDT to recover the data in physical space (f).
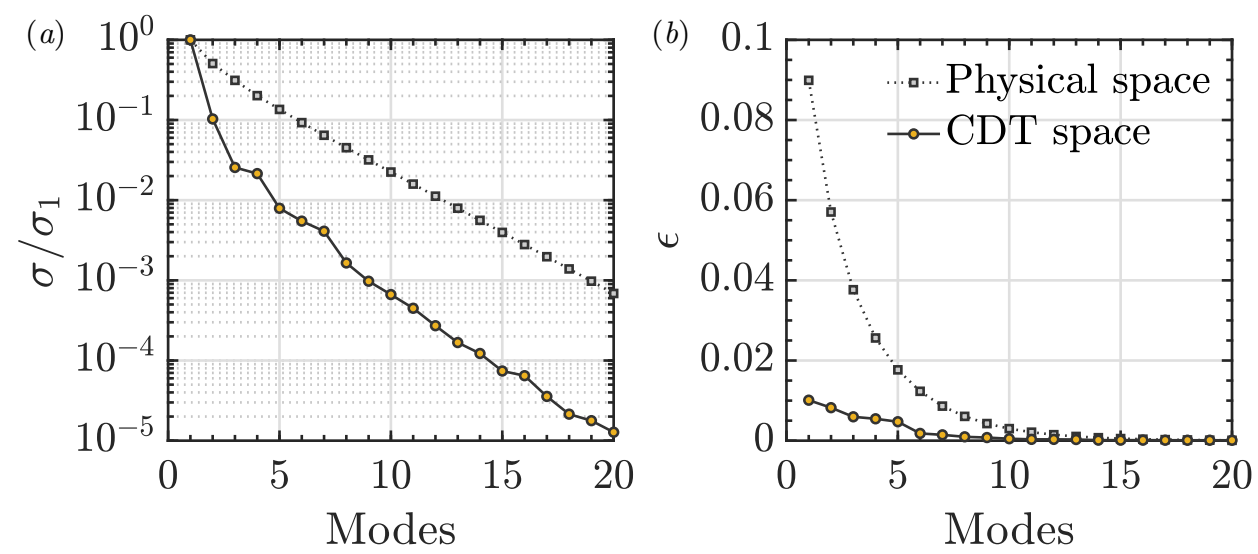

FIG. 4. Comparison of the singular value decay (a) and error of reduced order models (b) in the physical and CDT space.

to $-8 \leq x \leq 8$ with travel-in/out boundary conditions. The solution is obtained for $t \in[0,40]$ with a size of 268 grid points $\times 201$ time steps.

Figure 3(a) presents the evolution of the profile. The initial Gaussian soliton travels downstream with decaying amplitude. By the end of the time limit, part of the wave travelled out of the domain. Upon comparing the data (figure $3 \mathrm{~b}$ ) and its 2-mode ROM (figure $3 \mathrm{k}$ ) in the $x-t$ frame, it is evident that the ROM considerably distorted the data. On the contrary, the data in the CDT space (figure 3d) can be methodically reduced to two modes (figure 3e). The iCDT finally recovers the reduced data in the physical space (figure 3 ). There is hardly a discernible difference 
between the reconstructed data and its origin.

To quantify the performance of the ROM in CDT space, figure 4 plots the decay of singular values $\sigma$ (obtained from POD and normalised by the first value $\sigma_{1}$ ) and the error $\epsilon$ as functions of the number of modes. The singular value drop is much faster in the CDT space, implying reduced Kolmogorov n-width. The error has been defined as the root-mean-square level between the reconstructed data $u$ and ground-truth value $u_{0}$ :

$$
\epsilon=\sqrt{\frac{1}{N M} \sum_{i=1}^{N} \sum_{j=1}^{M}\left|u\left(x_{i}, t_{j}\right)-u_{0}\left(x_{i}, t_{j}\right)\right|^{2}} .
$$

Here $N$ and $M$ are the numbers of grid points and time steps. Figure 4 (b) indicates that the error is essentially reduced (up to $88 \%$ less) in particular in the regimes where fewer modes are kept.

\section{High dimensional problems: travelling Gaussian solitons}

Here we show the application of R-CDT space for higher-dimensional problems. The tested problems are the 2-D and 3-D travelling Gaussian solitons, given by

$$
u(x, y, z, t)=\exp \left[-a_{x}\left(x-\xi_{x}(t)\right)^{2}-a_{y}\left(y-\xi_{y}(t)\right)^{2}-a_{z}\left(z-\xi_{z}(t)\right)^{2}\right]
$$

The parameters $a_{x}, a_{y}, a_{z}$ determine the size of the soliton along each coordinate $(x, y, z)$. The time functions $\left(\xi_{x}(t), \xi_{y}(t), \xi_{z}(t)\right)$ control the centre of the soliton at time $t$. In the examples below, we have specified $a_{x}=a_{y}=-0.08$ for 2-D and $a_{x}=a_{y}=a_{z}=-0.05$ for 3-D problems.

In the $2 \mathrm{D}$ example, we demonstrate the method for random travelling with a non-differentiable trajectory. The domain is $(x, y)=[0,100] \times[0,100]$. The time functions $\xi_{x}(t), \xi_{y}(t), \xi_{z}(t)$ are randomly generated with value between 10 and 90. We show the instantaneous signal in figure 5(a), the R-CDT space is obtained by sequentially performing Radon (figure 5b) and CDT (figure 5k) transformation. We show the 4-mode ROM in figure 5d, iCDT in figure 5p and iRadon transform in figure 5 r recovering the physical space. To compare, we show the 25-mode (figure $5 \mathrm{~g}$ ) and 50-mode ROMs (figure 5 $\mathrm{h}$ ) built in the physical space. As can be seen, the random motions are hardly modelled in the physical space even with 50 modes. We show the singular value decay and the error in figure 6. For random motion problems, as can be seen here, the singular value drops very slow in the physical space. The error reduces almost linearly by increasing the number of modes. Besides, the proposed R-CDT space essentially outperforms the physical space also for 3-D problems. Figure 7 (a) visualises a Gaussian soliton travelling uniformly in a 3-D field. The singular values in R-CDT space reach a much faster drop (figure 7b), leading to smaller errors of ROM. The reconstructed data from the standard 4-mode ROM (figure 75) is compared with its counterpart in R-CDT space (figure $7 \mathrm{~d}$ ).

\section{DISCUSSION AND CONCLUDING REMARKS}

In this letter, we have proposed the Radon cumulative distribution transform (R-CDT) space for the model reduction of travelling waves up to three-dimensional problems. Contrary to physical space, the Kolmogorov n-width becomes sharply reduced in the R-CDT space. Accordingly, a more precise but smaller-sized reduced-order model becomes feasible. The strength of the method lies in its parameter-free and data-driven nature. Neither recognition of the physical process (e.g. detect travelling waves and obtain their velocities) nor data-training as usually required by machine learning is necessary. There is no limitation on the boundary condition since a spatial/temporal shift is not performed. The method is particularly outstanding for travelling dominated signals even with random trajectories. The weakness may emanate from the accuracy of the inverse transforms (iRadon and iCDT). When noise or more complex signals dominate, the error produced by a reduced-order model (ROM) in the R-CDT space may get amplified during the iCDT and iRadon process. This can be overcome by improving the algorithms of inverse transforms or combining more advanced techniques buildinwg the ROM. For a more complex data-set, e.g. coupled with turbulence, future extension of the method can focus on separating the travelling component and building reduced-order models, respectively.

The R-CDT space shares a broad junction to the state-of-art model reduction techniques. In this work, we have shown that even standard POD provides very efficient models in the R-CDT space. We hope this work creates a new ground for the model reduction of travelling wave problems. 

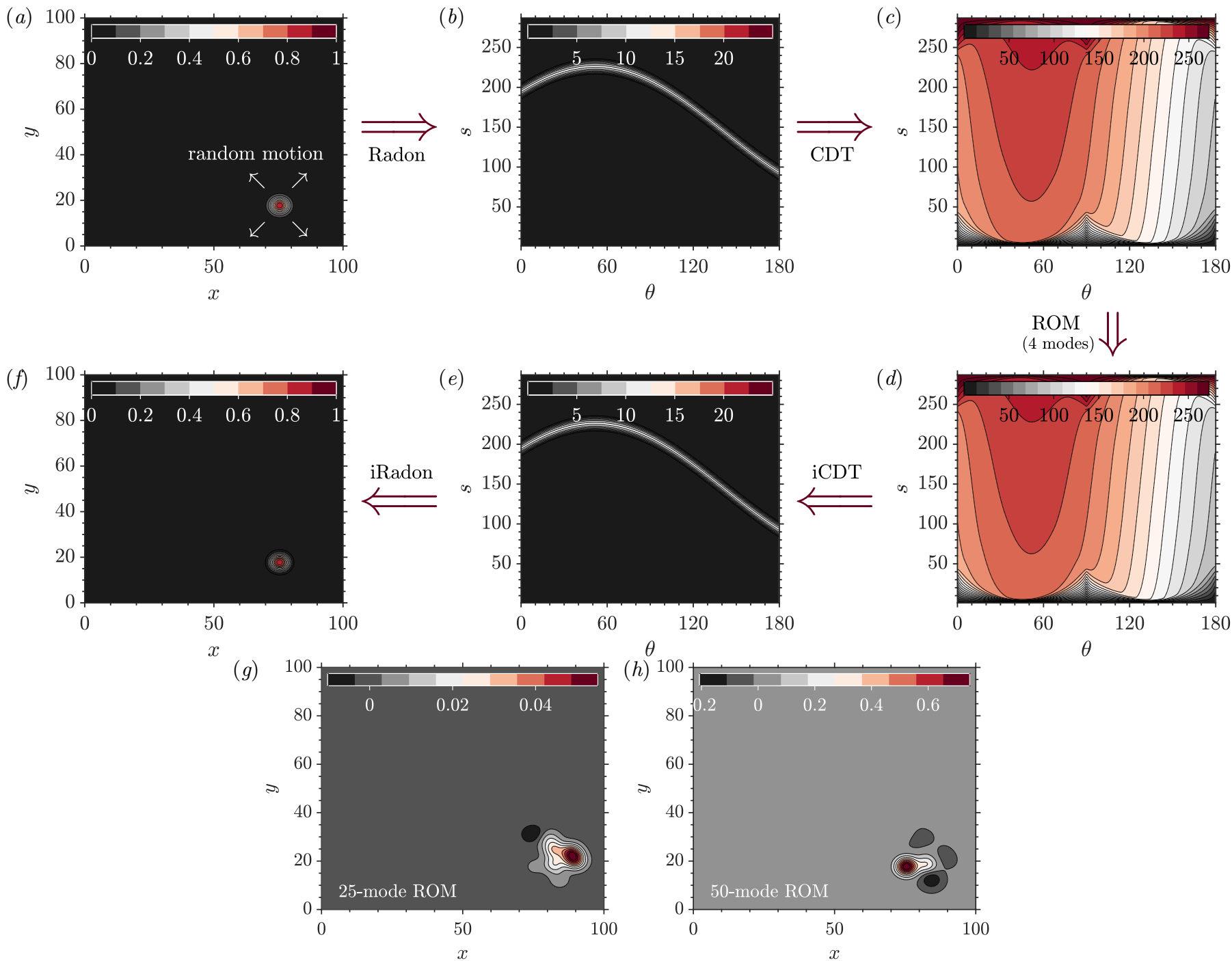

FIG. 5. The procedure to build a reduced-order model via R-CDT. The original signal (a) is transformed into the R-CDT space by sequentially applying the Radon transform (b) and CDT (c). The 4-mode ROM is built in the R-CDT space (d), this is followed by iCDT (e) and iRadon to recover the physical space (f). For comparison, the 25-mode and 50-mode ROMs built in the physical space are shown in panel $(\mathrm{g})$ and $(\mathrm{h})$. This figure shows a snapshot corresponding to $t=10$, a video covering the whole time horizon is available as a supplementary file.

This investigation is funded by the European Union's Horizon 2020 future and emerging technologies programme with agreement No. 828799. Calculations were performed on HPC-Midlands funded by the Engineering and Physical Sciences Research Council (grant number EP/K000063/1). J. R. acknowledges support from the Alexander von Humboldt Foundation. The authors would like to thank Professor Gustavo K. Rohde and Dr Hamidreza Eivazi for useful discussions.

\section{Appendixes}

Composition properties of CDT Let $g(x)$ be an invertible, differentiable function, the CDT of $f(g(x)) g^{\prime}(x)$ with respect to $r(x)$ is $\hat{f}_{g}(x)=g^{-1}(\hat{f}(x))$. The proof of this property follows the definition of CDT. From (2),

$$
\int_{x_{1}}^{\hat{f}(x)} f(u) \mathrm{d} u=\int_{x_{1}}^{\hat{f}_{g}(x)} f(g(u)) g^{\prime}(u) \mathrm{d} u=\int_{x_{1}}^{x} r(u) \mathrm{d} u .
$$



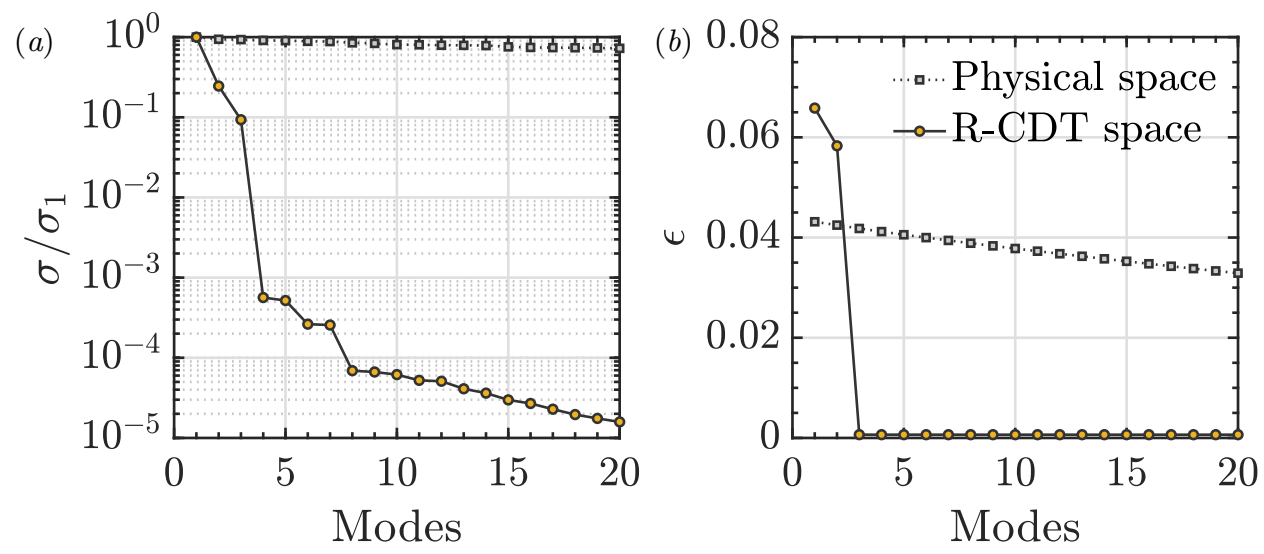

FIG. 6. Comparison of the singular value decay (a) and error of reduced order models (b) in the physical and R-CDT space.

(a)

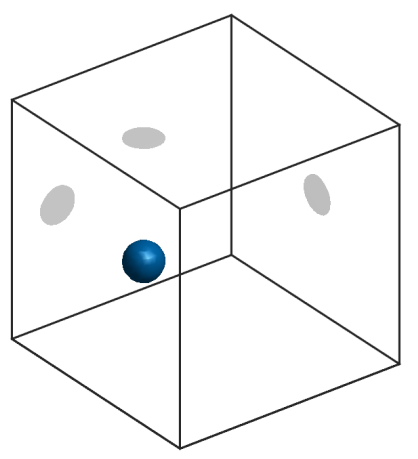

$(c)$

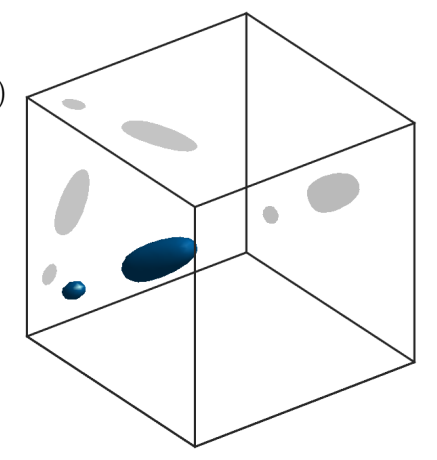

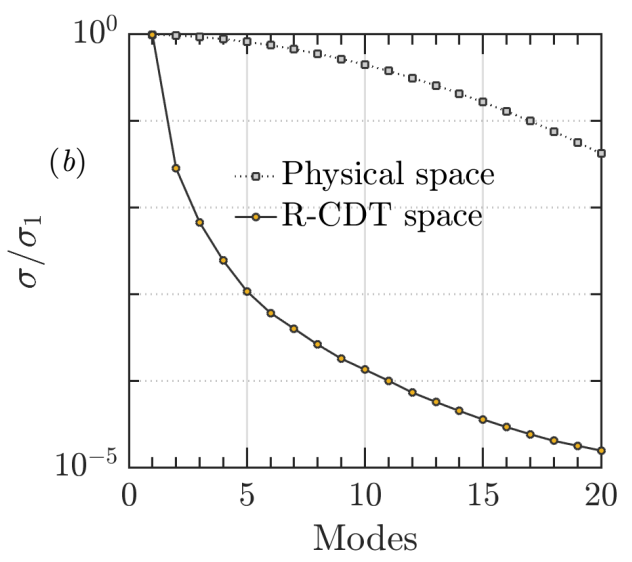

$(d)$

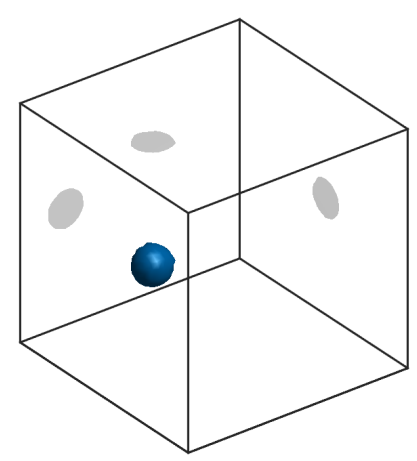

FIG. 7. The 3-D travelling Gaussian soliton visualised with an isosurface of $f(x, y, z, t)=0.05$ (a). Comparison of the singular value drop in the physical and R-CDT space (b). The reconstructed data from 4-mode ROM built in the physical (c) and R-CDT (d) spaces, respectively. Shadows in panel (a, c, d) indicate the projection of isosurfaces onto three coordinate planes. A movie showing the temporal evolution is available as a supplementary file. 
Let $g(u)=v$, then $g^{\prime}(u) \mathrm{d} u=\mathrm{d} v, 12$ becomes

$$
\int_{x_{1}}^{\hat{f}(x)} f(u) \mathrm{d} u=\int_{x_{1}}^{g\left(\hat{f}_{g}(x)\right)} f(v) \mathrm{d} v=\int_{x_{1}}^{x} r(u) \mathrm{d} u .
$$

Therefore,

$$
\hat{f}(x)=g\left(\hat{f}_{g}(x)\right) \Leftrightarrow \hat{f}_{g}(x)=g^{-1}(\hat{f}(x)) .
$$

From the composition property, let $g(x)=\alpha x-c t$, representing a travelling wave with phase velocity $c$ and scaling factor $\alpha$. Then the CDT of $f(\alpha x-c t)$ is given by $(\hat{f}(x)+c t) / \alpha$.

* maoxuerui@sina.com

[1] S. Kolouri, S. R. Park, and G. K. Rohde, The radon cumulative distribution transform and its application to image classification, IEEE transactions on image processing 25, 920 (2016).

[2] C. W. Rowley and S. T. Dawson, Model reduction for flow analysis and control, Annual Review of Fluid Mechanics 49, 387 (2017).

[3] S. L. Brunton, J. L. Proctor, and J. N. Kutz, Discovering governing equations from data by sparse identification of nonlinear dynamical systems, Proceedings of the national academy of sciences 113, 3932 (2016).

[4] C. W. Rowley and J. E. Marsden, Reconstruction equations and the karhunen-loève expansion for systems with symmetry, Physica D: Nonlinear Phenomena 142, 1 (2000).

[5] A. Mendible, S. L. Brunton, A. Y. Aravkin, W. Lowrie, and J. N. Kutz, Dimensionality reduction and reduced-order modeling for traveling wave physics, Theoretical and Computational Fluid Dynamics 34, 385 (2020).

[6] J. Reiss, P. Schulze, J. Sesterhenn, and V. Mehrmann, The shifted proper orthogonal decomposition: A mode decomposition for multiple transport phenomena, SIAM Journal on Scientific Computing 40, A1322 (2018).

[7] J. Sesterhenn and A. Shahirpour, A characteristic dynamic mode decomposition, Theoretical and Computational Fluid Dynamics 33, $281(2019)$

[8] D. Rim, S. Moe, and R. J. LeVeque, Transport reversal for model reduction of hyperbolic partial differential equations, SIAM/ASA Journal on Uncertainty Quantification 6, 118 (2018).

[9] N. Cagniart, Y. Maday, and B. Stamm, Model order reduction for problems with large convection effects, in Contributions to partial differential equations and applications (Springer, 2019) pp. 131-150.

[10] N. J. Nair and M. Balajewicz, Transported snapshot model order reduction approach for parametric, steady-state fluid flows containing parameter-dependent shocks, International Journal for Numerical Methods in Engineering 117, 1234 (2019).

[11] A. Iollo and D. Lombardi, Advection modes by optimal mass transfer, Physical Review E 89, 022923 (2014).

[12] B. Peherstorfer, Model reduction for transport-dominated problems via online adaptive bases and adaptive sampling, SIAM Journal on Scientific Computing 42, A2803 (2020).

[13] K. Lee and K. T. Carlberg, Model reduction of dynamical systems on nonlinear manifolds using deep convolutional autoencoders, Journal of Computational Physics 404, 108973 (2020).

[14] S. R. Park, S. Kolouri, S. Kundu, and G. K. Rohde, The cumulative distribution transform and linear pattern classification, Applied and Computational Harmonic Analysis 45, 616 (2018).

[15] G. Monge, Mémoire sur la théorie des déblais et des remblais, Histoire de l'Académie Royale des Sciences de Paris (1781).

[16] S. R. Deans, The Radon transform and some of its applications (Dover Publications, Inc., Mineola, New York, 2007).

[17] M. Shifat-E-Rabbi, X. Yin, A. H. M. Rubaiyat, S. Li, S. Kolouri, A. Aldroubi, J. M. Nichols, and G. K. Rohde, Radon cumulative distribution transform subspace modeling for image classification, arXiv preprint arXiv:2004.03669 (2020).

[18] N. Bonneel, J. Rabin, G. Peyré, and H. Pfister, Sliced and radon wasserstein barycenters of measures, Journal of Mathematical Imaging and Vision 51, 22 (2015).

[19] F. Bernard, A. Iollo, and S. Riffaud, Reduced-order model for the BGK equation based on POD and optimal transport, Journal of Computational Physics 373, 545 (2018). 